performed to determine differences between exposure variables (e.g. male versus female).

Results There were 36640 workers meeting inclusion criteria (average 305 cases per month; mean rate per month of 11.6 per 100000 working population). A total of 1,121,863 lost working weeks were compensated, with a median of 10 weeks. Thirty-two percent of cases did not have a record of attending hospital. The lowest median duration of time loss was among those involved in a train or tram crash (2.9 weeks) and the highest among those with quadriplegia (142.2 weeks). Duration of income support was significantly different within age, sex, injury type, severity, crash type, and compensation system groups.

Conclusions Results showed that RTC injuries of all severities caused considerable work absence. Measures of work absence can complement existing measures of RTC burden.

\section{O3B.2 OCCUPATIONAL EXPOSURES AND SICKNESS ABSENCE TRAJECTORIES AMONG FINNISH EMPLOYEES: A REGISTER LINKED NATIONALLY REPRESENTATIVE FOLLOW-UP STUDY}

\begin{abstract}
1,2Tea Lallukka*, 'Leena Kaila-Kangas, ${ }^{2,3}$ Minna Mänty, ${ }^{4}$ Johanna Seppänen, ${ }^{1}$ Eija Haukka, ${ }^{1}$ Johanna Kausto, ${ }^{1}$ Päivi Leino-Arjas, ${ }^{4}$ Risto Kaikkonen, ${ }^{1} J a a n a ~ H a l o n e n, ~ 1,2$ Rahman Shiri. ${ }^{1}$ Finnish Institute of Occupational Health, Helsinki, Finland; ${ }^{2}$ Department of Public Health, University of Helsinki, Helsinki, Finland; ${ }^{3}$ Department of Research, Development and Innovation (RDI), Laurea University of Applied Sciences, Vantaa, Finland; ${ }^{4}$ National Institute for Health and Welfare, Helsinki, Finland
\end{abstract}

\subsection{6/OEM-2019-EPI.63}

Background Physical workload factors have been linked to the risk of sickness absence, however, some work exposures can also be protective of work ability. We aimed to first identify sickness absence trajectories in a nationally representative occupational cohort, and second to examine the associations between physical workload factors, potential protective factors and sickness absence trajectories.

Methods We examined a nationally representative cohort of working aged Finnish adults interviewed and medically examined at baseline in 2000-2001 $(n=3814)$. Both potentially protective (sitting and keyboard use) and risk factors (e.g. handling of heavy loads and vibration) were included. All medically confirmed sickness absence periods 2002-2008 were included in the trajectory analyses to identify latent groups with similar sickness absence trends. These data were derived from the registers of the Social Insurance Institution of Finland. The models were adjusted for sociodemographic factors, health behaviors and physical and mental health.

Results We found a group with a very low level, a group with a stable low level, and a group with a high and increasing level of sickness absence. After full adjustments, prolonged sitting and use of keyboard emerged as protective factors that were associated with a lower risk of belonging to the trajectory with high and increasing sickness absence (HR 0.60; 95\% CI 0.45-0.80). In turn, the risk increased with the number of physical workload factors reported, and was highest for those with four or more workload factors (HR 2.84; 95\% 2.08-3.88).

Conclusions Physical work is associated with the risk of long-term sickness absence, with the highest risk found for those with several different physical workload factors. As the risk remained after controlling for various pertinent risk factors, this highlights the need to find ways to better maintain work ability of those with the physically most strenuous work.

\section{B.3 ESTIMATING THE IMPACT OF CHANGES TO OCCUPATIONAL STANDARDS FOR SILICA EXPOSURE ON LUNG CANCER MORTALITY}

${ }^{1}$ Alex Keil, 'David Richardson, ${ }^{1}$ Daniel Westreich, ${ }^{2}$ Kyle Steenland ${ }^{*} .{ }^{1}$ Rollins School of Public Health, Emory U, Atlanta, USA; ${ }^{2}$ Dept Epidemiology, UNC, Chapel Hill, US

\subsection{6/OEM-2019-EPI.64}

Background Respiratory exposure to silica is associated with the risk of death due to malignant and non-malignant disease. 2.3 million U.S. workers are exposed to silica. Occupational exposure limits for silica are derived from a number of lines of evidence, including observational studies. Observational studies may be subject to healthy worker survivor bias, which could result in underestimates of silica's impact on worker mortality and, in turn, bias risk estimates for occupational exposure limits.

Methods Using data on 65999 workers pooled across multiple industries, we estimate the impacts of several hypothetical occupational exposure limits on silica exposure on lung cancer and all-cause mortality. We use the parametric g-formula, which can account for healthy worker survivor bias.

Results Assuming we could eliminate occupational exposure, we estimate that there would be 20.7 fewer deaths per 1000 workers in our pooled study by age 80 (95\% confidence interval: 14.5, 26.8), including 3.91 fewer deaths due to lung cancer (95\% CI: 1.53, 6.30). Less restrictive interventions demonstrated smaller, but still substantial risk reductions.

Conclusions Our results suggest that occupational exposure limits for silica can be further strengthened to reduce silicaassociated mortality and illustrate how current risk analysis for occupational limits can be improved.

\section{O3B.5 THE MENTAL HEALTH OF POLICE, FIREFIGHTERS AND PARAMEDICS IN THE UK BIOBANK: A COMPARISON WITH THE GENERAL WORKING POPULATION}

Sharon Stevelink*, Nicola Fear, Matthew Hotopf. King's College London, London, UK

\subsection{6/OEM-2019-EPI.65}

It is our responsibility to protect and look after the health of members of the emergency services as this directly impacts on the country's readiness to respond to these disasters and is a critical part of our duty of care towards this important group of workers. This study examined the mental health outcomes and associations with individual and job characteristics among emergency services personnel compared to a random sample of working people, thereby using data from the UK Biobank. This data source contains data on over half a million adults in the UK, who were at the time of recruitment between 40 69 years. Over 280000 reported being in work. Current emergency services personnel were identified based on Standard Occupational Classification (SOC) 2000 codes. A random sample of age and sex matched people working in other occupations were selected from the UK Biobank for comparative purposes. The prevalence of the outcomes of interest, based 